\title{
Role of prebiotics on the microbiote
}

\begin{abstract}
In December 2016, a panel of experts in microbiology, nutrition and clinical research was convened by the International Scientific Association for Probiotics and Prebiotics to examine the definition and scope of prebiotics.
\end{abstract}

Volume 8 Issue 3 - 2018

\author{
Richard Haddad \\ Hospital Saint Louis Lariboisiere, France
}

Correspondence: Richard Haddad, Specialist, Hospital Saint Louis Lariboisiere, France, Email richardhaddad92@gmail.com

Received: June 18, 2018 | Published: June 25, 2018

\section{Introduction}

The panel has updated the definition of a prebiotic: a substrate that is selectively used by host micro-organisms conferring a benefit to health. (Action on the Microbiota) This definition extends the concept of prebiotics to possibly include non-carbohydrate substances, applications to body sites other than the gastrointestinal tract, and various categories other than food.

a. Main advantage of prebiotics: Improving human health by modulating the microbiome is an evolutionary strategy that is part of a holistic and non holistic approach to the well-being of people.

b. Point of impact of prebiotics: The rich and diverse microbial ecosystems that inhabit the surfaces mucous and cutaneous

c. Mechanism of action of prebiotics: Capacity to move the composition and metabolic signatures s microbial populations

d. The first prebiotics: For 20 years fructans (fructo-oligosaccharides (FOS) and inulin) and galactans (Galacto-oligosaccharides or GOS) fall into this category, their effects acting by enrichment of Lactobacillus and / or Bifidobacterium spp.

e. The most used FOS and GOS currently dominate the prebiotic category

f. Method of study of prebiotics: high throughput sequencing, It improved 1. Our knowledge of the composition of the microbiota, 2. And allowed the identification of additional substances influencing colonization.

g. Prebiotic action sites: effective on intestine and extra-intestinal sites, directly or indirectly skin mouth, vagina...

The different aspects discussed in consensus group meeting on December 9, 2016 in London: the evolution of the term prebiotic

I. Effects and selectivity

II. Substrates that are prebiotics

III. Metabolism of prebiotics

IV. Benefits of the host

\section{Pets}

VI. Tips for producers, consumers and regulators.

The activities of ISAPP are determined by the Board of Directors, composed of world-wide academic scientists. Through its Industry Advisory Committee, ISAPP integrates industry scientists into its activities and raises funds to advance its mission. However, no input to this consensus group process was provided by members of the Industry Advisory Committee. ISAPP functions as an independent, objective and scientific voice for probiotic and prebiotic fields. In 2004, the definition of prebiotics was changed to "selectively fermented ingredients that allow specific changes, both in the composition and/ or activity of the gastrointestinal microflora that confer benefits on the well-being and health of the host "The definition of prebiotics in 2008. By the UN and FAO this group proposed to redefine prebiotics as a "non-viable food component conferring a health benefit to the host associated with microbiota modulation". ${ }^{1}$ Here, selective fermentation was removed as a criterion, but in doing so, the definition was criticized for not excluding antibiotics.

Gibson et al., ${ }^{2} 2$ years later, define the more restricted category of "food prebiotics" (definition 2010) as "a selectively fermented ingredient that causes specific changes in the composition and / or activity of the gastrointestinal microbiota", thus conferring benefits to the health host. In 2015, Bindels proposed that the specificity requirements be deleted on the basis of reports showing that several taxa, rather than particular species, were enriched by probiotics. Another definition of a prebiotic 2015-2016 as "an indigestible compound which, by its metabolism by micro-organisms in the intestine, modulates the composition and / or the activity of the intestinal microbiota, thus conferring a physiological effect beneficial to the host". (This definition limits prebiotics to interactions with gut microbiota only. The current ISAPP consensus group now proposes the following definition of a 2017 prebiotic: a substrate that is selectively used by host micro-organisms that confer a health benefit. Definition much broader and applies to the intestinal microbiota intestinal and extra-intestinal.

\section{Acknowledgments}

None.

\section{Conflicts of interest}

The author declares there is no conflict of interest.

\section{References}

1. Glenn R Gibson, Robert Hutkins, Mary Ellen Sanders, et al. Expert consensus document: The International Scientific Association for Probiotics and Prebiotics (ISAPP) consensus statement on the definition and scope of prebiotics. Nat Rev Gastroenterol Hepatol. 2017;14(8):491502.

2. Quote Microbiology Food Microbiology Microbiota Nutrition. 2017. 